\title{
Boundary limits of Green's potentials along curves
}

by

\author{
JANG-MEI GLORIA WU (Urbana; III.)
}

Abstract. On a disk, the boundary limits of the Green's potential along level curves of certain functions are zero almost everywhere. A similar problem on a Jordan domain with rectifiable boundary is also studied. These extend the results by Littlewood [2], Tolsted [4] and Arsove [1].

1. Introduction. Let $u$ be the Green's potential of a nonnegative mass distribution on $|z|<1$. Littlewood ([2], p. 391) proved that the radial limit of $u$ is zero at almost all points on $|z|=1$. For a fixed $\alpha$ in $(-\pi / 2, \pi / 2)$, Tolsted ([4], p. 640) showed that for almost all values of $\xi$, $u(z)$ tends to zero as $z$ tends to $e^{i \xi}$ along the line segment which makes an angle $\alpha$ with the radius at $e^{i \xi}$. Zygmund ([4], pp. 644-645) pointed out that the nontangential limit of a Green's potential need not exist at any point on $|z|=1$. Therefore, it is natural to ask for curves $l(\xi)$ in $|z|<1$ approaching $e^{i \xi}, 0 \leqslant \xi<2 \pi$, so that we have $\lim u(z)=0$ as $\approx$ tends to $e^{i \xi}$ along $l(\xi)$ for almost all $\xi$. The main result, Theorem 2 , gives a broad family of curves, namely the level curves of a certain kind of function. A similar result in Jordan domain with rectifiable boundary is also obtained, which extends a theorem by Arsove ([1], p. 267) on a very special domain of this type.

All the results can be applied to the subharmonic functions with positive harmonic majorant instead of Green's potentials, with the aid of the Riesz decomposition theorem.

The author wishes to thank Robert Kaufman for some helpful conversations and the referee for pointing out a related paper on the "nontagential $L^{p}$ convergence" of Green's potential by Ziomek [6].

2. Limits on the disk. From now on, we use $s, \sigma$ to denote $1-|z|$ and $1-|w|$, respectively, whenever $z, w$ are points in the complex plane. Sets on $|z|=1$ are identified with sets on $(-\pi, \pi]$ whenever convenient. We use $I$ to denote an arc of $|z|=1$ in $\operatorname{Re} z>0$ and identify it with $[a, b]$. We use $\mu$ to denote a nonnegative mass districution on $|w|<1$ satisfying

$$
\int_{|w|<1} \sigma d \mu(w)<\infty
$$


and $u$ to denote the Green potential of $\mu$ defined by

$$
u(z)=\int_{|w|<1} G(z, w) d \mu(w)
$$

where $G$ is the Green function on $|z|<1$. The symbols $M$ and $m$ denote strictly positive constants independent of $u ; M$ and $m$ may vary from one line to another.

THEOREM 1. Suppose $\frac{1}{2}<e<1$. Let $l(\xi)$ be a Jordan curve in $e \leqslant|z|$ $\leqslant 1$ and $\operatorname{Re} z>0$ joining $e^{i \xi}$ to $|z|=0$ if $\xi \in I$ and let $\Omega$ be the closed region bounded by $l(a), l(b), I$ and $|z|=c$. Suppose $\bigcup_{I} l(\xi)=\Omega$ and whenever $z, w \in \Omega, z$ on $l(\xi)$, w on $l(\xi+\tau)$, we have

(i) $\quad|z-w| \leqslant M|| z|-| w||$ if $\tau=0$,

(ii) $|z-w| \geqslant m|\tau|$, and

(iii) $\quad|z-w| \leqslant M|\tau|$ if $|z|=|w|$.

Then $\lim u(z)=0$ as $z \rightarrow e^{i \xi}$ along $l(\xi)$ for almost all $\xi$ on $I$.

Proof. The value of Green's function is given by

$$
G(z, w)=\log \frac{|1-\bar{z} w|}{|z-w|}
$$

It can be proved ([2], pp. 384-385) that

$$
G(z, w) \leqslant \frac{M \sigma s}{|z-w|^{2}} .
$$

We shall give further estimates for $G(z, w)$.

From (ii) and (iii) we see that two different curves are disjoint and no two points on the same curve have the same moduli. Fixing $z$, we locate $w$ by the coordinates $(\sigma, \tau)$; recall $\sigma=1-|w|$. We divide $\Omega$ into three sets in terms of $w$ as follows:

$$
\begin{aligned}
& R_{1}: c \leqslant|w| \leqslant 1,|\tau| \geqslant s, \\
& R_{2}: c \leqslant|w| \leqslant 1,|\tau|<s,|\sigma-s| \geqslant s / 2, \\
& R_{3}: c \leqslant|w| \leqslant 1,|\tau|<s,|\sigma-s|<s \mid 2 .
\end{aligned}
$$

We require

$$
\begin{array}{ll}
G(z, w) \leqslant \frac{M \sigma s}{\tau^{2}} & \text { on } R_{1}, \\
G(z, w) \leqslant \frac{M \sigma s}{s^{2}+\tau^{2}} \quad \text { on } R_{2}, \\
G(z, w) \leqslant \frac{\sigma}{s} \log \frac{M s^{2}}{\tau^{2}} \text { on } R_{3} .
\end{array}
$$

In fact, on $R_{1}$ we have, from (2.4) and (ii),

$$
G(z, w) \leqslant \frac{M \sigma s}{|z-w|^{2}} \leqslant \frac{M \sigma s}{m^{2} \tau^{2}} .
$$

On $R_{2}$, with the aid of (2.4) we have

$$
G(z, w) \leqslant \frac{M \sigma s}{|z-w|^{2}} \leqslant \frac{M \sigma s}{|z|-|w|^{2}}=\frac{M \sigma s}{|\sigma-s|^{2}} \leqslant \frac{4 M \sigma s}{s^{2}}<\frac{8 M \sigma s}{s^{2}+\tau^{2}} .
$$

If $w$ is on $R_{3}$, let $p$ be the point on $l(\xi)$ of the same modulus as $w$. Thus from (i) and (iii) we see that

$$
\begin{aligned}
|z-w| & \leqslant|z-p|+|p-w| \leqslant M|| z|-| p||+M|\tau| \\
& =M|\sigma-s|+M|\tau| \leqslant M s .
\end{aligned}
$$

Also we observe $|1 / \bar{z}-z| \leqslant 4 s$ because $|z| \geqslant 1 / 2$. Therefore, from (2.3) and $|\sigma-s| \leqslant s / 2$, we have

$$
G(z, w) \leqslant \frac{1}{2} \log \frac{|1 / \bar{z}-w|^{2}}{|z-w|^{2}} \leqslant \frac{\sigma}{s} \log \frac{M s^{2}}{m^{2} \tau^{2}} .
$$

We have proved (2.5)-(2.7).

The following part of the proof is a slight variant of Littlewood's proof for the radial limits; we shall not write too much detail and refer the reader to [2].

Let $\varepsilon\left(\varrho_{0}\right)$ be $\int \sigma d \mu(w)$ taken over $\Omega \cap\left\{\varrho_{0} \leqslant|w|<1\right\}\left(\equiv \Omega\left(\varrho_{0}\right)\right)$ and $\eta\left(\varrho_{0}\right)=\sqrt{\varepsilon\left(\varrho_{0}\right)}$. From (2.1) we have

$$
\lim _{\varrho_{0} \rightarrow 1} \eta\left(\varrho_{0}\right)=0 \text {. }
$$

Let $\Phi(q)$ be $\int \sigma d \mu(w)$ taken over $\Omega\left(\varrho_{0}\right) \cap\{a \leqslant \tau \leqslant q\}$. Then $0 \leqslant \Phi^{\prime}(q)$ $\leqslant \eta\left(\varrho_{0}\right)$ in a set $E\left(\varrho_{0}\right) \subset(a, b)$ of measure at least $b-a-\eta\left(\varrho_{0}\right)$. It is enough to prove

$$
\lim \sup \int_{\Omega\left(\varrho_{0}\right)} G(z, w) d \mu(w) \leqslant M \eta\left(\varrho_{0}\right)
$$

as $z \rightarrow e^{i \xi}$ along $l(\xi)$ for all $\xi$ in $E\left(\varrho_{0}\right)$ because

for all $\xi$ in $(a, b)$.

$$
\limsup _{z \rightarrow e^{i \xi}} \int_{\{|z|<1\} \backslash \Omega\left(e_{0}\right)} G(z, w) d \mu(w)=0
$$

Let $\xi \in E\left(\varrho_{0}\right)$; we may assume $\xi=0$. Let $d=\max \{-a, b\}, t$ in $[0, d]$ and $J(t)$ be $\int \sigma d \mu(w)$ taken over $\Omega\left(\varrho_{0}\right) \cap\{|\tau| \leqslant t\}$. By the characteristic property of $E$,

$$
\limsup _{t \rightarrow 0} \frac{J(t)}{t} \leqslant 2 \eta\left(\varrho_{0}\right) .
$$


Littlewood showed that nonnegative, monotone increasing function $J$ on $[0, d]$ with property (2.9) must satisfy:

$$
\begin{gathered}
\limsup _{s \rightarrow 0} s \int_{s}^{d} \frac{d J(\tau)}{\tau^{2}} \leqslant M \eta\left(\varrho_{0}\right), \\
\limsup _{s \rightarrow 0} s \int_{0}^{s} \frac{d J(\tau)}{s^{2}+\tau^{2}} \leqslant M \eta\left(\varrho_{0}\right),
\end{gathered}
$$

and

$$
\limsup _{s \rightarrow 0} \frac{1}{s} \int_{0}^{s} \log \frac{M s^{2}}{\tau^{2}} d J(\tau) \leqslant M \eta\left(\varrho_{0}\right) .
$$

Now (2.8) follows easily from the above three inequalities and the estimates for Green's functions (2.5)-(2.7). Theorem 1 is proved.

CoRorlary. Suppose $f$ is a real-valued $U^{1}$ function on $|z| \leqslant 1$ and $\partial f / \partial \theta \neq 0$ on $I$. Then for almost all $\xi$ in $I, \lim u(z)=0$ as $z \rightarrow e^{i \xi}$ along the level curve ending at $e^{i \xi}$.

Proof. Let $V$ be a neighborhood of $I$ such that $|\partial f| \partial \theta \mid \geqslant m$ on $V \cap\{|z|<1\}$. From the implicit function theorem, there is a simple $O^{1}$ level curve $l(\xi)$ from $\partial V$ to $e^{i \xi}$ whenever $\xi \epsilon I$. Choo $\theta e$ between $\frac{1}{2}$ and 1 so that $|z|=c$ meets each $l(\xi)$; and the closed region $\Omega$ bounded by $l(a)$, $l(b), I$ and $|z|=c$, is contained in $V$. Because $\partial f / \partial \theta$ is of one sign in $V$, each point in $\Omega$ is on some $l(\xi), \xi \in I$; moreover on $l(\xi),|d \theta / d r|<M$ uniformly for $\xi \epsilon I$. By the inequality $|d \theta| d r \mid<M$, (i) in Theorem 1 holds. that

Assume $|\nabla f|<M$ on $\Omega$ and $z \epsilon l(\xi) \cap \Omega, w \epsilon l(\xi+\tau) \cap \Omega$. We observe

$$
M|z-w| \geqslant|f(z)-f(w)|=\left|f\left(e^{i \xi}\right)-f\left(e^{i(\xi+\tau)}\right)\right| \geqslant m|\tau| .
$$

Thus (ii) in Theorem 1 is true.

Assume, moreover, $|z|=|w|$. With the aid of $|\partial f| \partial \theta \mid \geqslant m$, we have

$$
m|z-w| \leqslant|f(z)-f(w)|=\left|f\left(e^{i \xi}\right)-f\left(e^{i(\xi+\tau)}\right)\right| \leqslant M|\tau| .
$$

Thus (iii) in Theorem 1 is true. Corollary follows from Theorem 1 .

We shall consider limits of $u$ along level curves of a function, required only to be $C^{1}$ within triangles at the boundary points.

If $z$ is on the boundary of a domain $D$, by a triangle at $z$ wo mean a closed triangle in $\bar{D}$ with a vertex at $z$.

THEOREM 2. Let $E$ be a set of positive measure on $|z|=1$ and $f$ be $C^{1}$ in $|z|<1$. Suppose for each $\theta$ in $E, f$ and $\nabla f$ can be extended continuously to $e^{i \theta}$ through a triangle $T(\theta)$ at $e^{i \theta}, \nabla f\left(e^{i \theta}\right) \neq(0,0)$ and is not normal to $|z|=1$. Then for almost all $\theta \in E$, there is a unique level curve $l(\theta)$ of $f$ ending at $e^{i \theta}$ nontangential to the unit circle and $\lim u(z)=0$ as $z \rightarrow e^{i \theta}$ along $l(\theta)$
Here we regard two curves ending at $e^{i \theta}$ the same if they coincide in a neighborhood of $e^{i \theta}$. And we do not exclude the existence of tangential leviel sets at $e^{i \theta}$.

Proof. We first give an outline of the argument. We shall reduce to the special case: $E$ is closed, $f$ and $\nabla f$ are continuous on $\bigcup_{E} T(\theta)$ and all the $T(\theta)$ 's are identical and symmetric about the radius at $e^{i \theta}$. Then we shall construct a saw-toothed region $R$ in $|z|<1$ with teeth at $E$, on $\bar{R}$ we have uniform Taylor expansion of $f$ and the lengths of subarcs of $\partial R$ have some nice property. The idea of this construction is from Zygmund ([7], Vol. II, pp. 199-201) and E. M. Stein ([3], pp. 201-203). Finally, we shall modify the function $f$ outside $\bar{R}$ and show the level curves of the new $f$ satisfy the conditions in Theorem 1 .

Step I. By reducing $T(\theta)$ if necessary, we assume that the two sides emanating from $e^{i \theta}$ are of rational lengths, the two angles between these sides and the radius at $e^{i \theta}$ are rational multiples of $\pi$. By splitting. $E$ into a denumerable family of subsets, we may reduce $E$ to the case in which all the $T(\theta)$ 's, $\theta \in E$, are identically situated. From Egoroff's theorem, we may also assume that $E$ is closed and $f$ and $\nabla f$ are uniformly continuous on $\bigcup_{\theta \in E} T(\theta)(\equiv S)$. Let $a(\theta) \epsilon[0, \pi / 2)$ be the angle between $\nabla f\left(e^{i \theta}\right)$ and the tangent at $e^{i \theta}$ for $\theta \in E$ and $\beta$ be a rational multiple of $\pi$ such that $\pi / 2>\beta>\max \left\{\alpha(\theta)+\frac{3}{4}(\pi / 2-\alpha(\theta)): \theta \in E\right\}$. For each point of density $\theta$ of $E$ we may find a small triangle $T^{*}(\theta)$ symmetric about the radius at $e^{i \theta}$, with angle $2 \beta$ at $e^{i \theta}$ and $T^{*}(\theta) \subseteq S$. Since almost all points in $E$ are points of density, by considering a subset of points of density and replacing $T(\theta)$ by $T^{*}(\theta)$ if necessary, we may assume that $E$ is closed, each $T(\theta)$ is symmetric about its radius, with angle $2 \beta$ at $e^{i \theta}$ and $f, \nabla f$ are uniformly continuous on $\bigcup T(\theta)$. As before, we may also assume all the $T(\theta)$ 's are identical.

Step II. Let $R=\bigcup_{E} R(\theta)$, where $R(\theta)$ is the region bounded by the two tangent lines from $e^{i \theta}$ to the circle $|z|=\sin \beta$ and by the more distant arc of $|z|=\sin \beta$ between the points of contact. Since $\bar{R}(\theta)$ and $T(\theta)$ coincide near $e^{i \theta}, f$ and $\nabla f$ are continuous on $\bigcup_{E} \bar{R}(\theta)(=\bar{R}$ because $E$ is closed).

Several facts concerning $R$ will be observed in succession. (a) $\bar{R}$ is star-like with respect to the origin. (b) If $z, w \in \bar{R}$, then there exists a point $q$ in $\bar{R}$ such that the line segments $\overline{z, q}, \overline{q, w}$ are in $\bar{R}$ and $|z-q|+|q-w|$ $\leqslant M|z-w|$; here $M$ depends on $\beta$. To see this, choose $q=z$ if $\overline{z, w} \subseteq \bar{R}$, otherwise choose $q$ to be the point of the largest modulus so that $\overline{z, q}$ and $\overline{q, w}$ are in $\bar{R}$. (c) If $z, w \in \bar{R}$, then $f(w)-f(z)=(w-z) \cdot \nabla f(z)+o(|w-z|)$ and "o" depends only on $|z-w|$. This is a simple consequence of $(\mathbf{b})$. 
(d) The total length of the subarc of $\partial R$ in $\xi \leqslant \theta \leqslant \xi+\tau$ is at most $M \tau$ and at least $m \tau(0 \leqslant \tau \leqslant 2 \pi)$.

Step III. Because $\bar{R}$ is star-like with respect to the origin 0 , we may define $f_{1}$ on $|z| \leqslant 1$ as follows: if $z \epsilon \bar{R}, f_{1}(z)=f(z)$; if $z \notin \bar{R}, f_{1}(z)=$ the value of $f$ at the intersection of $\partial R$ and the radius through $z$. We shall consider the level curves of $f_{1}$.

For any fixed $\theta \epsilon E$, let $V$ be a neighborhood of $e^{i 0}$ disjoint from $|z|<\sin \beta$ and, if $z \in V \cap \bar{R}$, then $|\nabla f(z)| \geqslant m$ and the angle between $\nabla f(z)$ and $\overline{z, 0}$ is greater than $\frac{3}{4}(\pi / 2-\alpha(\theta))$. For $z, w \epsilon \partial R \cap V$, the angle between $\overline{z, w}$ and $\overline{0, z}$ is at least $\beta$, which is greater than $\alpha(\theta)+\frac{3}{4}(\pi / 2-\alpha(\theta))$, thus the angle between $\nabla f(z)$ and $\overline{z, w}$ is less than $\pi / 4+\alpha(\theta) / 2$, which is bounded away from $\pi / 2$. Therefore through each point on $\partial R \cap V$, there is a level curve of $f_{1}$ in $V$, composed of an arc in $R \cap V$ except the end points and a radial segment outside $R$. Let us assume $e^{i \theta}$ is in the right half plane. We choose a short arc $I$ of $|z|=1$ containing $e^{i \theta}$ and a number $c$ close to 1 in $\left(\frac{1}{2}, 1\right)$ so that for every $\xi \in I$ there is a level curve $l(\xi)$ of $f_{1}$ joining $e^{i \xi}$ to $|z|=c$, the closed region. $\Omega$ bounded by $l(a), l(b)$, $|z|=c$ and $I$, is contained in $\nabla$, and finally, whenever $z, w \in \Omega$,

$$
|0(|w-z|)|<\frac{1}{2}|z-w| \cos \left(\frac{\pi}{4}+\frac{\alpha(\theta)}{2}\right) \min _{V \cap \bar{R}}|\nabla f| .
$$

We claim that $|f(w)-f(z)| \geqslant m|w-z|$ if $z, w \in \partial R \cap \Omega$. In fact, from (c) in Step $I I$ and the angle between $\nabla f(z)$ and $\overline{z, w}$ we have

$$
\begin{aligned}
|f(w)-f(z)| & \geqslant|(w-z) \cdot \nabla f(z)|-|o(|w-z|)| \\
& \geqslant|w-z||\nabla f(z)| \cos \left(\frac{\pi}{4}+\frac{\alpha(\theta)}{2}\right)-|o(|w-z|)| \\
& \geqslant m|w-z|,
\end{aligned}
$$

where $m$ is a constant dependent on $\theta$.

Now we shall show (i)-(iii) in Theorem 1. (i) is clear because $\left|\frac{d \theta}{d r}\right|$ is uniformly bounded on level curves in $\bar{R} \cap \Omega$ and the level curves are radial segments in $\Omega \backslash \bar{R}$. Let $z \epsilon l(\xi) \cap \Omega, w \in l(\xi+\tau) \cap \Omega$ and $z_{0}, w_{0}$ be the intersections of $\partial R$ with $l(\xi), l(\xi+\tau)$, respectively. We assume $|\partial f| \partial \theta|\geqslant m, M \geqslant| \nabla f \mid \geqslant m$ on $\Omega \cap \bar{R}$. To prove (ii), if $z, w \notin R$, it is clear from the construction of $f_{1}$ that $|z-w| \geqslant m|\tau| ;$ if $z$ and $w$ are in $\bar{R}$, then with the aid of (c) in Step II and the inequality in the last paragraph we have

$$
M|z-w| \geqslant|f(z)-f(w)|=\left|f\left(z_{0}\right)-f\left(w_{0}\right)\right| \geqslant m\left|z_{0}-w_{0}\right| \geqslant m|\tau| ;
$$

if one is in $R$, the other is outside $\bar{R}$; by breaking $\overline{z, w}$ into two pieces, we conclude $|z-w| \geqslant m|\tau|$. Thus (ii) holds.
To prove (iii), we assume $|z|=|w|$. Clearly, $|z-w| \leqslant M|\tau|$ if $z$ and $w$ are outside $R$. If $z$ and $w$ are in $\bar{R}$ and the circular arc $\overline{z, w}$ centered at 0 is completely in $\bar{R}$, then with the aid of $|\partial f| \partial \theta|\geqslant m, M \geqslant| \nabla f \mid$ on $\Omega \cap \bar{R}$ and (c), (d) we see that

$$
m|z-w| \leqslant|f(z)-f(w)|=\left|f\left(z_{0}\right)-f\left(w_{0}\right)\right| \leqslant M\left|z_{0}-w_{0}\right| \leqslant M|\tau| .
$$

In general, we may always write $\widehat{z, w}$ as sum of arcs with end points falling into the above two types, therefore $|z-w| \leqslant M|\tau|$. Thus (iii) is true.

As a consequence of Theorem 1 , for almost all $\xi$ in $I, \lim u(z)=0$ as $z \rightarrow e^{i \xi}$ along $l(\xi)$, where $I$ is an arc containing $e^{i \theta}$. Because the above fact is true for every $\theta$ in $E, E$ is closed under reduction and the nontangential level curves of the $f$ are the same as those of $f_{1}$ at $E$, Theorem 2 follows.

Remark. A shorter proof can be based on Whitney's extension theorem. Since $f$ is $C^{\mathrm{I}}$ in $R$ and $f(w)-f(z)=(w-z) \cdot \nabla f(z)+o(|w-z|)$ whenever $z, w \in \bar{R}$ Whitney ([3], p. 194, 4.7 or [5] p. 65) showed that $f \mid \bar{R}$ can be extended to a function $C^{1}$ on the entire plane. A simple application of the Corollary of Theorem 1 gives Theorem 2. We note that the function $f_{1}$ is much simpler to construct but not smooth.

3. Jordan domains. Let $A$ be a Jordan domain with rectifiable boundary and $\varphi$ be a conformal mapping from $|z|<1$ to $A$. It is known ([7], Vol. I, Chap. VII, $\S 10$ ) that (a) $\varphi$ can be extended to a homeomorphism of $|z| \leqslant 1$, (b) $\partial \varphi\left(z_{0}\right) / d \theta$ exists and is different from zero at almost every point $z_{0}$ on the unit circle, call these points $S$, (c) for each $z_{0} \in S$, $i z \varphi^{\prime}(z)$ tends to $\partial \varphi\left(z_{0}\right) / \partial \theta$ as $z \rightarrow z_{0}$ nontangentially and $\partial A$ has a tangent at $p\left(z_{0}\right),(\mathrm{d})$ if $\Gamma$ is a curve approaching $z_{0} \in S$ from $|z|<1$, intersecting with the horizontal line through $z_{0}$ at an angle $\alpha$, then the image $\varphi(\Gamma)$ intersects the horizontal line through $\varphi\left(z_{0}\right)$ at an angle $\alpha+\operatorname{Arg}\left[\frac{1}{i z_{0}} \frac{\partial \varphi}{\partial \theta}\left(z_{0}\right)\right]$, (e) sets of measure zero on $|z|=1$ correspond to sets of measure zero on $\partial A$, and vice versa. On $A$ we have the following theorem:

Thmomen 3. Let $F$ be a subset of $\phi(S)$ of positive linear measure. Suppose $g$ is $C^{1}$ in $A, g$ and $\nabla g$ can be extended continuously to $K^{\mathrm{r}}$ through triangles at $F$, moreover $\nabla g \neq(0,0)$ and is not normal to $\partial A$ at any point in II. If $v$ is a Green's potential, not identically $+\infty$, on $A$, then for almost all $z_{0}$ on $F, \lim v(z)=0$ as $z \rightarrow z_{0}$ along the level curve of $g$ ending at $z_{0}$ nontangential to $\partial A$.

Proof. Under $\varphi^{-1}$, the triangles at $F$ become curvilinear triangles at $\varphi(F)(\equiv E)$. To each point $z \in E$ we introduce a triangle $T(z)$ in the corresponding curvilinear triangle. 
Let $f=g \circ \varphi$ and write $\varphi=u+i v$. Clearly, $f$ is $C^{1}$ in $|z|<1$. Because

$$
\nabla f=\left(g_{x}(\varphi) u_{x}+g_{y}(\varphi) v_{x}, g_{x}^{\prime}(\varphi) u_{y}+g_{y}(\varphi) v_{y}\right)
$$

from (c) we see that $f$ and $\nabla f$ are continuous up to $E$ through triangles $T(z)$ 's. From (b), (c), (d) and the Cauchy-Riemann equations, we have $\nabla f \neq(0,0)$ and is not normal to the unit circle. Since the level sets are preserved under conformal mappings, we conclude Theorem 3 from Theorem 2.

ExAMPLES. (1) The following result of Arsove ([1], p. 267) is a simple consequence of Theorem 3 and the Riesz decomposition theorem: if $h$ is a subharmonic function on $\{|z|<1,|z-1|<\delta\}$ with positive harmonic majorant then $\lim h\left(\mathrm{re}^{i \theta}\right)$ exists for almost all $e^{i \theta}$ in $\{|z|<1,|z-1|<\delta\}$. $r \rightarrow 1$.

(2) $A$ and $v$ are defined as in Theorem 3. Then at almost all points of $\partial A$, where the tangents of $\partial A$ are not horizontal, $v$ has horizontal limit zero.

\section{References}

[1] M. G. Arsove, The Lusin-Privalov theorem for subharmonic functions, Proc. London Math. Soc. (3), 14 (1964), pp. 271-298.

[2] J. E. Littlew ood, On functions subharmonio in a circle (II), ibid. (2), 28 (1928), pp. $383-394$.

[3] E. M. Stein, Singular integrals and differentiability properties of funotions, Princeton, 1970 .

[4] E. B. Tolsted, Limiting values of subharmonic funotions, Proc. Amer. Math. Soc. 1 (1950), pp. 636-647.

[5] H. Whitney, Analytic extensions of differentiable functions defined in olosed sets, Trans. Amer. Math. Soc. 36 (1934), pp. 63-89.

[6] L. Ziomek, On the boundary behavior in the metric $L^{p}$ of subharmonic funotions, Studia Math. 29 (1967), pp. 97-105.

[7] A. Zygmund, Trigometric series, Vol. I and II, Cambridge, 1959.

\section{UNIVERSITYY OF ITUINOTS}

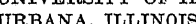

\section{Multipliers and unconditional Schauder bases in Besov spaces}

\section{by}

\author{
HANS TRIEBEL (Jena)
}

Abstract. The paper contains: (a) Explicit representations for distributions belonging to Besov spaces, (b) A system of analytic functions which is an unconditional Schauder basis in Besor spaces, (c) A necessary and sufficient algebraic condition for multipliers in Besov spaces, (d) Remarks on embedding theorems for Besov spaces.

1. Introduction. The paper deals with isotropic Besov spaces $B_{p, q}^{s}$ ( = Lipschitz spaces $\Lambda_{p, q}^{s}$ ) defined in $\boldsymbol{R}_{n}$, where $-\infty<s<\infty ; 1<p<\infty$; and $1 \leqslant q \leqslant \infty$. There exists a large variety of different characterizations of distributions belonging to these spaces [3]-[7]. A summary may be found in [9], Chapter 2. In Section 2 of this paper a new representation formula is given. As an immediate consequence there is obtained a common unconditional Schauder basis in all the spaces $B_{p, q}^{s}$ (provided that $q<\infty)$, consisting of entire analytic functions of exponential type, Section 3. A second application of the representation formula yields a necessary and sufficient condition for multipliers in $B_{p, q}^{s}$, Section 4 . A more detailed discussion of this result will be given later on. Section 5 contains remarks on embedding theorems.

2. Representations. $\boldsymbol{R}_{n}$ denotes the $n$-dimensional real Euclidean space. The general point in $\boldsymbol{R}_{n}$ is denoted by $x=\left(x_{1}, \ldots, x_{n}\right)$. $S\left(=S\left(\boldsymbol{R}_{n}\right)\right)$ is the usual Schwartz space of all complex-valued infinitely differentiable rapidly decreasing functions, defined on $\boldsymbol{R}_{n}$. As usual, $S^{\prime}\left(=S^{\prime}\left(\boldsymbol{R}_{n}\right)\right)$ is the space of tempered distributions, the dual space to $S$. The Fourier transform in $S^{\prime}$ is denoted by $F$, its inverse by $F^{-1}$. If $f \in S$, then

$$
(F f)(\xi)=(2 \pi)^{-\frac{n}{2}} \int_{\mathbf{R}_{n}} e^{-i x \xi} f(x) d x,
$$

where $x \xi=\sum_{j=1}^{n} x_{j} \xi_{j}$. If $-i$ is replaced by $i$, then one obtains the corresponding formula for $\mathrm{F}^{-1}$.

Use the following decomposition of $\boldsymbol{R}_{n}$ : Let $\sigma=\left(\sigma_{1}, \ldots, \sigma_{n}\right)$ be a vector in $\boldsymbol{R}_{n}$, where each of the components $\sigma_{j}$ is either \pm 1 or \pm 3 , 